\title{
Drug delivery for tuberculosis: is inhaled therapy the key to success?
}

\author{
Daniela Traini $^{\star 1}$ \& Paul M Young ${ }^{1}$ \\ ${ }^{1}$ Respiratory Technology, Woolcock Institute of Medical Research, Sydney, NSW 2037 \& Discipline of Pharmacology, Sydney \\ Medical School, University of Sydney, NSW 2006, Australia \\ *Author for correspondence: Daniela.traini@sydney.edu.au
}

"The questions on the utility of inhaled TB therapy are still open-ended, but in a world where the risk of untreatable infections is ever-present, the need for continued research into TB, perhaps focusing on 'game changers' such as inhaled therapies, are of utmost importance."

First draft submitted: 10 April 2017; Accepted for publication: 24 April 2017; Published online: 25 September 2017

Keywords: inhaled therapy $\bullet$ Mycobacterium tuberculosis $\bullet$ tuberculosis

\section{Drug delivery for tuberculosis}

Worldwide, tuberculosis (TB) has $86 \%$ treatment success rate in new cases, leaving more than 1 million new patients without a cure [1]. Thus, the need for both shorter treatment regimens and new antibiotics is great. Current oral or injectable standard anti-TB drug regiments are well established and relatively inexpensive therapies. Still, it is evident from the rise of multidrug resistance TB (MDR-TB) worldwide, that among other factors, these approaches are not preferred among patients and healthcare professionals and, have been proven to have varying degrees of success.

In the past few years there has been an enormous interest in developing more effective anti-TB vaccines [2,3], developing new anti-TB drugs that shortens treatment and exploiting the genome to accelerate the diagnosis and treatment of drug-resistant TB [4,5]. Importantly, there has also been a rising interest in developing anti-TB therapies via inhalation [6,7]. This is because, used as primary or adjunct to the current standard therapy for TB, inhaled therapy may hold the key as a potential new delivery mode that has improved health benefits. These are likely to include reduced treatment times and possibly limited insurgence of drug resistance. Specifically, for inhaled TB treatment, drugs should target alveolar macrophages that harbor microorganisms and/or maintain high drug concentration at the infection site in the lung [8]. Inhaled drug delivery offers this prospect and has the potential to achieve high concentrations of pharmacologic agents in the lungs, while producing adequate systemic concentrations through alveolar-capillary absorption to treat extrapulmonary sites of infection. It would seem that a localized delivery method would be intuitively attractive for a disease that is very often restricted, but not limited, to the lung.

\section{Inhalation therapy for TB}

Inhalation therapy for TB is not a 'new' approach and began in the late 1940s when streptomycin started showing signs of resistance [9]. Since then, many different formulation approaches have been investigated. Micro [10,11], nanoparticles [12,13], liposomes [14], in situ forming liposomes [15], proliposomes [16,17] and liposphere [18], with single [19,20] and combination drugs [21,22], have all been manufactured with different degrees of success, although mostly limited to data collected in vitro or from preclinical studies where, for the majority, formulations were found effective in reducing bacterial counts. Nevertheless, there is no completed clinical study and it is difficult to identify which system would be the most advantageous to take further.

Recent setbacks have also hindered research advances in this field, that is, the suspension of enrolment in STAND (Phase III combination trial with new drug pretomanid) [23], the discontinuation of candidate TBA-354 (due to 
signs of toxicity) [24] and the official end of development of AZD5847 (due to lack of anti-TB activity) [25], adding more uncertainties to the future of research in this area.

Thus, despite the large number of research investigations into different formulations, trials testing combinations of drugs already on the market and some good clinical news such as the start of Phase I clinical trial for Qurient's Q203 [26] (representing a new drug class and a new sponsor in TB clinical trial), it is surprising to see this apparent 'defiance' in translation of inhaled therapies for TB to allow clinical studies to be initiated or new drug classes to be developed.

There are many known advantages to inhaled therapy for TB. These include: reduced dosage (estimated up to ten-times less used for the standard oral treatment of care); limited side effects (e.g., liver and renal toxicity and peripheral neurotoxicity) with the same level of efficacy specifically targeted to macrophages that harbor the bacilli; and shortened treatment times with cheaper cost and improved patient compliance. Furthermore, existing treatment regimens already approved by the WHO with anti-mycobacterial activity but which cannot be given systematically due to high toxicity, could be used if repurposed for the inhalation route. In the same way, big pharma is interested in developing inhaled antibiotics for other pathologies, paving the way for inhaled therapies for TB.

Still, there are disadvantages of inhaled therapy that cannot be ignored, including the possibly that oral therapy could still be needed in conjunction to inhaled therapy to reach the granulomas with sufficient systemic doses, since $15 \%$ of TB cases involves extrathoracic disease. However, computational studies based on rifapentine and isoniazid inhaled formulation have predicted that antibiotic concentrations in granulomas remain more stable over an entire dosing window (2 weeks) with inhaled formulations than with daily oral doses [27]. Furthermore, it is doubtful whether aerosol formulations will achieve sufficient therapeutic levels in the serum; and reducing the clearance rate of the drug from the lung without reducing the free concentration will still need to be achieved, losing the advantage of local delivery. Additionally, other challenges remain: for instance, the use of specialized delivery devices could, perhaps, make this therapeutic avenue more expensive then oral delivery, resulting in a disadvantage for implementing this therapy on a large scale, especially in developing countries, considering the stringent current pharmacoeconomic environments.

Currently, large risks are deemed acceptable in MDR-TB due to the greater unmet need and potential for patient benefit. Consequently, since inhaled clinical trials for MDR-TB are outside our reach with the limited funds available and limited industrial support, should researchers try to drive discoveries further by expediting preclinical work? This would allow viable candidates into clinical studies and, consequently, allow to assess the validity of inhaled therapies in cases where other options are no longer available, based on expanded access (compassionate use). These advances will have a significant impact on patient care and health policy for TB and infectious diseases in general. This area is already being explored with the creation of the special medical use and adaptive licensing pathways for registration of new treatments for drug-resistant infections based on small clinical trials. Potentially this could replace the requirement for conventional Phase III trials, transforming TB therapy.

\section{The future of TB therapy via inhalation?}

In conclusion, while infection control and early detection remain the foundation for reducing TB exposure among noninfected individuals, and the current ambitious TB goals for 2030 are helping in stepping up the response against $\mathrm{TB}$, there is still the need to ask few questions: are new drugs or new delivery routes really necessary to stop $\mathrm{TB}$ or should we concentrate efforts on new vaccines and/or on measuring an individual's transcriptional signature to allow for a more personalized monitoring of the disease? Moreover, should clinicians be convinced in trialing the inhalation route for TB or asked to use more judiciously currently available oral and intravenous therapies to reduce MDR-TB cases while still waiting for a cure, or at least mitigate the global burden of TB?

The questions on the utility of inhaled TB therapy are still open-ended, but in a world where the risk of untreatable infections is ever-present, the need for continued research into TB, perhaps focusing on 'game changers' such as inhaled therapies, are of utmost importance.

Financial \& competing interests disclosure

The authors have no relevant affiliations or financial involvement with any organization or entity with a financial interest in or financial conflict with the subject matter or materials discussed in the manuscript. This includes employment, consultancies, honoraria, stock ownership or options, expert testimony, grants or patents received or pending, or royalties.

No writing assistance was utilized in the production of this manuscript. 


\section{References}

1 WHO. Global tuberculosis report 2016. (2016). www.who.int/tb/publications/global_report/en

2 Ottenhoff TH, Kaufmann SH. Vaccines against tuberculosis: where are we and where do we need to go? PLoS Pathog. 8(5), e1002607 (2012).

3 Weiner J, 3rd, Kaufmann SH. Recent advances towards tuberculosis control: vaccines and biomarkers. J. Intern. Med. 275(5), 467-480 (2014).

4 Lechartier B, Rybniker J, Zumla A, Cole ST. Tuberculosis drug discovery in the post-post-genomic era. EMBO Mol. Med. 6(2), 158-168 (2014).

5 Phelan J, Coll F, Mcnerney R et al. Mycobacterium tuberculosis whole genome sequencing and protein structure modelling provides insights into anti-tuberculosis drug resistance. BMC Med. 1431 (2016).

6 Misra A, Hickey AJ, Rossi C et al. Inhaled drug therapy for treatment of tuberculosis. Tuberculosis (Edinb) 91(1), 71-81 (2011).

7 Pham DD, Fattal E, Tsapis N. Pulmonary drug delivery systems for tuberculosis treatment. Int. J. Pharm. 478(2), 517-529 (2015).

8 Du Toit LC, Pillay V, Danckwerts MP. Tuberculosis chemotherapy: current drug delivery approaches. Respir. Res. 7 118 (2006).

9 Davies J, Davies D. Origins and evolution of antibiotic resistance. Microbiol. Mol. Biol. Rev. 74(3), 417-433 (2010).

10 Durham PG, Zhang Y, German N et al. Spray dried aerosol particles of pyrazinoic acid salts for tuberculosis therapy. [corrected]. Mol. Pharm. 12(8), 2574-2581 (2015).

11 Gupta A, Pant G, Mitra K, Madan J, Chourasia MK, Misra A. Inhalable particles containing rapamycin for induction of autophagy in macrophages infected with Mycobacterium tuberculosis. Mol. Pharm. 11(4), 1201-1207 (2014).

12 Wang C, Hickey AJ. Isoxyl aerosols for tuberculosis treatment: preparation and characterization of particles. AAPS PharmSciTech 11(2), 538-549 (2010).

13 Ahmad MI, Ungphaiboon S, Srichana T. The development of dimple-shaped chitosan carrier for ethambutol dihydrochloride dry powder inhaler. Drug Dev. Ind. Pharm. 41(5), 791-800 (2015).

14 Chimote G, Banerjee R. In vitro evaluation of inhalable isoniazid-loaded surfactant liposomes as an adjunct therapy in pulmonary tuberculosis. J. Biomed. Mater. Res. B 94b(1), 1-10 (2010).

15 Gaur PK, Mishra S, Gupta VB, Rathod MS, Purohit S, Savla BA. Targeted drug delivery of rifampicin to the lungs: formulation, characterization, and stability studies of preformed aerosolized liposome and in situ formed aerosolized liposome. Drug Dev. Ind. Pharm. 36(6), 638-646 (2010).

16 Rojanarat W, Nakpheng T, Thawithong E, Yanyium N, Srichana T. Inhaled pyrazinamide proliposome for targeting alveolar macrophages. Drug Deliv. 19(7), 334-345 (2012).

17 Patil-Gadhe AA, Kyadarkunte AY, Pereira M et al. Rifapentine-proliposomes for inhalation: in vitro and in vivo toxicity. Toxicol. Int. 21(3), 275-282 (2014).

18 Singh C, Koduri LV, Dhawale V et al. Potential of aerosolized rifampicin lipospheres for modulation of pulmonary pharmacokinetics and bio-distribution. Int. J. Pharm. 495(2), 627-632 (2015).

19 Chan JG, Duke CC, Ong HX et al. A novel inhalable form of rifapentine. J. Pharm. Sci. 103(5), 1411-1421 (2014).

20 Chan JGY, Tyne AS, Pang A et al. Murine pharmacokinetics of rifapentine delivered as an inhalable dry powder. Int. J. Antimicrob. Ag. 45(3), 319-323 (2015).

21 Chan JGY, Chan HK, Prestidge CA, Denman JA, Young PM, Traini D. A novel dry powder inhalable formulation incorporating three first-line anti-tubercular antibiotics. Eur. J. Pharm. Biopharm. 83(2), 285-292 (2013).

22 Chan JGY, Tyne AS, Pang A et al. A rifapentine-containing inhaled triple antibiotic formulation for rapid treatment of tubercular infection. Pharm. Res-Dordr. 31(5), 1239-1253 (2014).

23 Dawson R, Diacon AH, Everitt D et al. Efficiency and safety of the combination of moxifloxacin, pretomanid (PA-824), and pyrazinamide during the first 8 weeks of antituberculosis treatment: a Phase $2 \mathrm{~b}$, open-label, partly randomised trial in patients with drug-susceptible or drug-resistant pulmonary tuberculosis. Lancet 385(9979), 1738-1747 (2015).

24 Alliance T. Phase 1 clinical trial of TB drug candidate TBA-354 discontinued 2017 (2016).

25 Furin J, Du Bois J, Van Brakel E. Early bactericidal activity of AZD5847 in pulmonary tuberculosis. Presented at: 46th Union World Conference on Lung Health. Cape Town, South Africa, 2-6 December 2015.

26 Pethe K, Bifani P, Jang J et al. Discovery of Q203, a potent clinical candidate for the treatment of tuberculosis. Nat. Med. 19(9), 1157-1160 (2013).

27 Linderman JJ, Cilfone NA, Pienaar E, Gong C, Kirschner DE. A multi-scale approach to designing therapeutics for tuberculosis. Integr. Biol. (Camb.) 7(5), 591-609 (2015). 
